\title{
Methylmalonic acid as an indicator of vitamin B12 deficiency in patients on metformin
}

\author{
Norbert Shtaynberg, Manjinder Singh, Phillip Sohn, Michael Goldman ${ }^{*}$, Neil Cohen
}

Staten Island University Hospital, Afghanistan; ${ }^{*}$ Corresponding Author: mikeygoldman@yahoo.com

Received 28 May 2011; revised 27 July 2011; accepted 26 December 2011

\section{ABSTRACT}

Context: Metformin is frequently prescribed for the treatment of type 2 diabetes mellitus. It is recommended as a first line agent by the American Diabetes Association. Vitamin B12 deficiency has been suggested as a side effect of metformin therapy; however, previous studies have not assessed the utility of methylmalonic acid levels as an indicator of vitamin B12 status. Objective: To investigate the prevalence of vitamin B12 deficiency in patients on metformin therapy for diabetes by utilizing both vitamin B12 and methylmalonic acid levels. Design, Setting, and Patients: Eighty-eight patients with diabetes, who were either on or off metformin therapy for at least thirty days, were enrolled in a case-controlled study. Blood work and questionnaires were used for analysis. Main Outcome Measures Study: Aims were to detect a clinically significant difference in the prevalence of vitamin B12 deficiency between metformin users and non-users, where such deficiency is defined by both low vitamin B12 and elevated methylmalonic acid levels. Results: Two Sample Equal Variance T-Tests were used to compare averages of measured values and the Chisquare test was used to determine the significance of calculated vitamin B12 deficiency rates between the two groups of patients. Two separate methods for defining vitamin B12 deficiency were utilized. There was no difference in the prevalence of vitamin B12 deficiency in metformin users compared with non-users by either method. Average homocysteine levels were higher in those not on metformin therapy. Conclusion: Vitamin B12 deficiency as defined by an elevated methylmalonic acid level was no greater in patients with diabetes on metformin therapy versus those patients not on metformin treatment.
Keywords: Metformin; Vitamin B12; Methylmalonic Acid; Diabetes Mellitus

\section{INTRODUCTION}

There are 23.6 million people in the United States with diabetes mellitus (approximately $8 \%$ of the total population), and $90 \%-95 \%$ of those have Type 2 diabetes. Currently, the yearly cost burden of diabetes is estimated to be roughly $\$ 174$ billion, and if current trends continue, one-third of the population will eventually develop diabetes. In 2006, diabetes was the sixth leading cause of death in America [1].

One of the standard treatments for type II diabetes is metformin. Clinical trials such as the United Kingdom Prospective Diabetes Study have shown the long-term benefits of metformin in decreasing diabetic-associated complications, and metformin has become the most prescribed oral hypoglycemic agent in the United States. However, metformin is not without adverse reactions. While gastrointestinal side effects are well-known, the link between metformin use and vitamin B12 deficiency is not as well recognized. Previous studies have suggested a prevalence of vitamin B12 deficiency of up to $30 \%$ in patients taking metformin [2,3]. Adams, et al. showed vitamin B12 levels returned to normal in half the patients who stopped taking the medication [3]. Metformin may affect vitamin B12 absorption by altering the secretion of intrinsic factor [4]. Dosage and chronicity of use of metformin have been studied as possible factors impacting vitamin B12 status [3].

Elevated serum levels of homocysteine and methylmalonic acid have been shown to be more sensitive markers of vitamin B12 deficiency than serum B12 concentrations alone. However, unlike hyperhomocysteinemia, elevated methylmalonic acid is also highly specific for vitamin B12 deficiency. Some studies have shown slight elevations of homocysteine levels among patients taking metformin [4,5]. However, the correlation between metformin use and methylmalonic acid concentrations has not been well studied. 


\section{MATERIALS AND METHODS}

\subsection{Subjects}

Patients aged 20 - 80 years with a history of type 2 diabetes who presented to Staten Island University Hospital or its' primary care clinic were screened for inclusion in the study. Each patient was questioned about his or her diabetes history and medication use. Patients had to have been on or off metformin treatment for a minimum of 30 days to be considered for the study. Patients were excluded if they were already on vitamin B12 replacement therapy or had a known history of vitamin B12 or folate deficiency. Other exclusion criteria included a history of hematological cancer, methotrexate use, pernicous anemia, alcohol intake, adherence to a vegetarian diet, gastric surgery, and/or inflammatory bowel disease. Patients on insulin therapy were excluded as well. 88 patients met criteria and consented to participate in the study -47 were taking metformin alone or in combination with another oral hypoglycemic agent, and 41 were on other oral hypoglycemic agents and had not taken metformin in the last 30 days. Each patient's chart was reviewed to determine the dosage of metformin prescribed.

The study was approved by the hospital's IRB committee and all patients provided written informed consent.

\subsection{Laboratory Investigations}

Patients had their blood drawn at the time of an office visit in the primary care clinic or within 24 hours of presentation to the hospital (to avoid alteration of laboratory data in response to hospital diabetes management). Vitamin B12, homocysteine, methylmalonic acid (MMA), serum creatinine, complete blood count (CBC), and glycosylated hemoglobin (HgA1C) levels were obtained. Vitamin B12, MMA, and homocysteine levels were analyzed using immunoassay on the DXI by Beckman, a GC-MS analyzer, and a Chemiluminescent Immunoassay System on the Immulite by Johnson and Johnson respectively. Standardized data collection forms were used to record the data for analysis.

\section{STATISTICAL METHODS}

This was a case-control study to determine the prevalence of Vitamin B12 deficiency in patients on metformin therapy for Type II Diabetes.

The following criteria were used to assess Vitamin B12 deficiency:

1) Vitamin B12 < 180 or $180<$ Vitamin B12 < 300 with Methylmalonic acid $>318$

(B12/MA Criteria)

OR:
2) Methylmalonic acid >318 (MA Only Criterion)

All analyses were conducted separately for each criterion for B12 deficiency. All tests were performed at $\alpha=$ 0.05 .

Differences in the distributions between metformin users $(n=47)$ and non-metformin users $(n=41)$ in B12 deficiency for categorical variables were assessed using Fisher's exact test. Fisher's exact test was also used to evaluate relationships between dose (for metformin users only) and demographic characteristics (Age, Sex, Race) and the prevalence of B12 deficiency. Dose was dichotomized as " $\leq 1000 \mathrm{mg}$ " and ">1000 mg". The Mantel-Haenszel test was used to investigate the relationships of sex and age to Vitamin B12 deficiency, while controlling for metformin use.

Differences between Vitamin B12 deficient subjects and non-deficient subjects by Homocysteine, $\mathrm{CBC}$, Hemoglobin $\mathrm{A} 1 \mathrm{C}$, and Creatinine levels were measured using the Wilcoxon rank sum test. Logistic regression analysis was used to assess the potential confounding effects of diabetes and A1C blood levels. Multiple logistic regression models were used to evaluate the relationship between Vitamin B12 deficiency (B12/MA Criteria) and metformin use and Vitamin B12 deficiency (using both criteria) with the prevalence of anemic subjects.

\section{RESULTS}

\subsection{Analysis for B12/MA Criteria}

There were 41 control subjects and 47 case subjects.

The prevalence of B12 deficiency did not differ significantly between groups (taking metformin vs. not taking). The prevalence rates were $17.1 \%$ and $12.8 \%$, respectively (Table 1)

Among the 47 metformin users, the daily dosage was $1000 \mathrm{mg}$ or less in 27 subjects and more than $1000 \mathrm{mg}$ in 20 subjects. For these subjects the rates of deficiency were $14.8 \%$ and $10.0 \%$, respectively, which was not significantly different (Table 2).

There was a significant association between age and B12 Deficiency $(\mathrm{p}<0.031)$ (Table 3). The prevalence of

Table 1. Prevalence of B12 deficiency based on both criteria.

\begin{tabular}{cccc}
\hline & Vit B12 Deficient & Not Vit B12 Deficient & P \\
\hline Criterion 1 & 17.07 & 12.77 & 0.76 \\
Criterion 2 & 31.71 & 19.15 & 0.22 \\
\hline
\end{tabular}

Table 2. B12 deficiency in relation to metformin dosing.

\begin{tabular}{cccc}
\hline & $\leq 1000 \mathrm{mg}$ & $>1000 \mathrm{mg}$ & $\mathrm{P}$ \\
\hline Criterion 1 & 14.81 & 10 & 1 \\
Criterion 2 & 14.81 & 25 & 0.47 \\
\hline
\end{tabular}


Table 3. Various factors and their association with B12 deficiency, evaluated seperately for each Criterion.

\begin{tabular}{cccccc}
\hline & & \multicolumn{2}{c}{ Criterion 1 } & \multicolumn{2}{c}{ Criterion 2 } \\
\hline \multirow{2}{*}{ Sex } & Male & 16.67 & $\mathrm{P}=0.76$ & 22.92 & $\mathrm{P}=0.63$ \\
& Female & 12.5 & & 27.5 & \\
& $<51$ & 9.09 & $\mathrm{P}=0.03$ & 22.73 & $\mathrm{P}=0.79$ \\
& $51 \leq 62$ & 4.55 & & 18.18 & \\
Age & $62 \leq 75$ & 34.78 & & 30.43 & \\
& $75+$ & 9.52 & & 28.57 & \\
& Non White & 6.67 & $\mathrm{P}=0.45$ & 13.33 & $\mathrm{P}=0.34$ \\
Race & White & 16.44 & & 27.4 & \\
\hline
\end{tabular}

B12 deficiency was low and relatively constant in the younger three age groups; in the $62 \leq 75$ age group, the prevalence is significantly higher.

No other variables were significantly associated with metformin use. In particular, Table 4 shows means for laboratory parameters according to deficiency status, regardless of metformin usage. None of these parameters differed significantly between deficient and non-deficient subjects.

All logistic regression models failed to demonstrate metformin use or B12 deficiency as significant predictors of anemia. ( $p=0.93$ and $p=0.17$ respectively).

\subsection{Analysis for MA-Only Criterion (Criterion 2)}

There were 41 non-metformin subjects and 47 metformin subjects.

For the primary question, the prevalence of B12 deficiency did not differ significantly between groups (met- formin no vs. yes). The prevalence rates were $31.7 \%$ and $9.2 \%$, respectively (Table $\mathbf{1}$ ).

Among the 47 metformin users, the daily dosage was $1000 \mathrm{mg}$ or less for 27 subjects and more than $1000 \mathrm{mg}$ for 20 subjects. For these subjects the rates of deficiency were $14.8 \%$ and $25.0 \%$, respectively, which were not significantly different (Table 2).

Unlike for the B12/MA criteria (criteria 1), there was no association between age and deficiency rates (Table 3).

Hemoglobin, along with homocysteine counts, was significantly different between vitamin B12 deficient and non-deficient subjects via criterion 2 only. For Hemoglobin, the $\mathrm{p}$ value was $<0.042$, and for homocysteine, $\mathrm{p}<$ 0.0003 .

\section{DISCUSSION}

Previous studies have indicated that there is a correlation between the use of metformin, vitamin B12 deficiency, and elevated homocysteine levels. However, the role of MMA has not been well studied. In this study, where elevated MMA levels were used to categorize vitamin B12 deficiency, a correlation between metformin use and B12 deficiency was not observed. Of note, two patients in each group had normal MMA levels in the presence of abnormally low serum vitamin B12 levels $(<180 \mathrm{pg} / \mathrm{ml})$. Of 22 patients with low normal vitamin B12 concentrations $(180-300 \mathrm{pg} / \mathrm{ml})$, only 6 had elevated MMA levels. Alternatively, of 22 patients with elevated MMA levels, only 9 had vitamin B12 concentrations $<300 \mathrm{pg} / \mathrm{ml}$. If MMA is considered a more sensitive test for clinically relevant vitamin B12 deficiency [6], then the prevalence of such deficiency may have been underestimated in previous studies. When we analyzed our data we used a subset of patients who have

Table 4. Lab parameter means and their significance between deficient and non deficient for both methods.

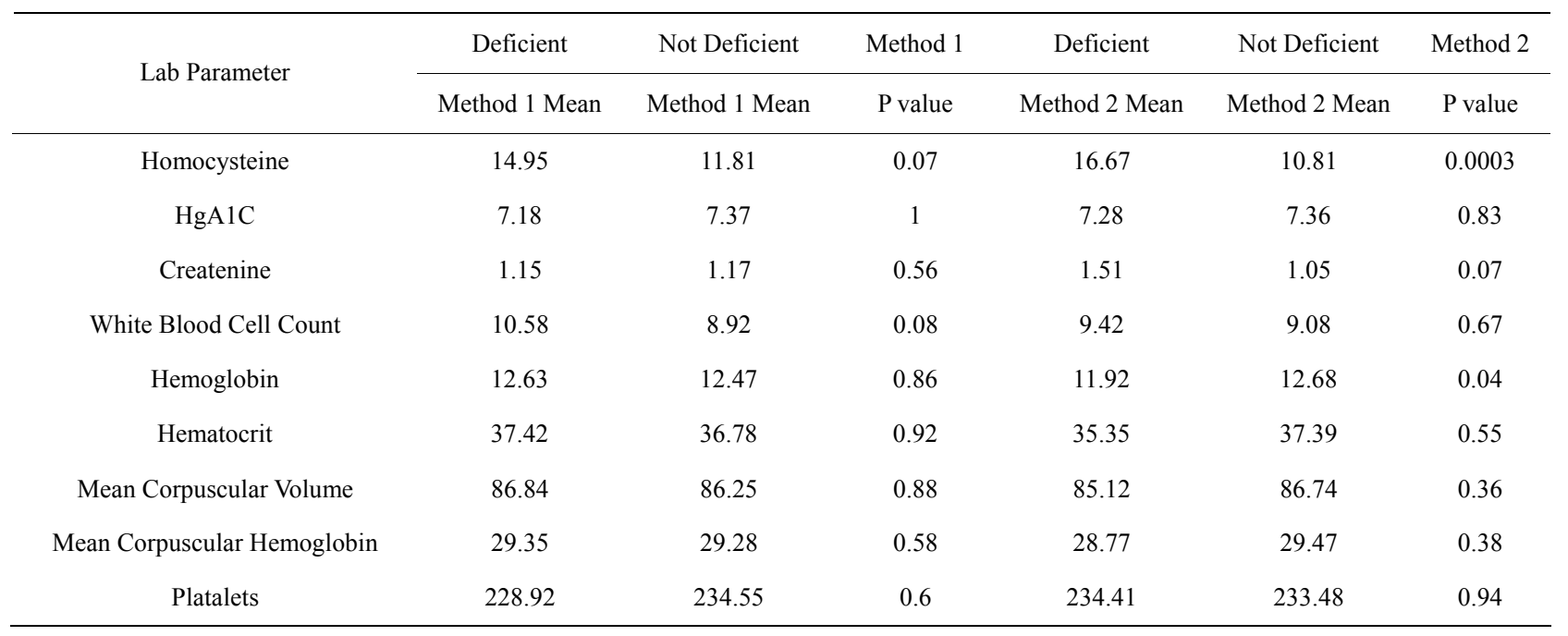


high MMA levels (>318 nmol/L) regardless of B12 levels and found no difference in vitamin B12 deficiency. Likewise, an overestimation of true B12 deficiency may occur when measured with B12 levels, as MMA is highly specific for ruling out disease. The serum vitamin $\mathrm{B} 12$ concentration is known to fluctuate more readily than MMA after alterations in eating habits and transfusions along with other factors; further, serum vitamin B12 levels may not reflect intracellular B12 concentrations [7].

Greater homocysteine concentrations were observed in the patients not on metformin therapy, in contrast to a prior study (4). However, this large, randomized-controlled trial included patients treated with metformin while they were on insulin treatment; the significance of which is unknown. Other smaller studies have demonstrated little or no effect of metformin treatment on homocysteine levels [5,8-11]. In the current study, possible confounding factors including familial hyperhomocysteinemia, inflammation, dietary factors other than vegetarianism; as well, documented folate deficiency may have affected homocysteine levels.

Additional limitations of this study include a small sample size, lack of information of other confounding variables such as duration of therapy and dosage, and absence of signs and symptoms of vitamin B12 deficiency.

In concordance with current guidelines, MMA levels should be used in combination with vitamin B12 levels to determine vitamin B12 status. Thus, additional studies examining B12 status in diabetes patients should utilize MMA measurements. Larger, randomized-controlled trials utilizing MMA are needed to better assess the risk of metformin therapy and development of clinically relevant vitamin B12 deficiency.

\section{REFERENCES}

[1] Centers for Disease Control and Prevention (CDC) (2009) Diabetes: Successes and opportunities for populationbased prevention and control: At a glance 2009. http://www.cdc.gov/nccdphp/publications/aag/ddt.htm

[2] Tompkins, G.H., Hadden, D.R., Weaver, J.A., et al. (1971) Vitamin B12 status of patients on long-term metformin therapy. British Medical Journal (BMJ), 2, 685-687. doi:10.1136/bmj.2.5763.685

[3] Adams, J.F., Clark, J.S., Ireland, J.T., et al. (1983) Malabsorption of vitamin B12 and intrinsic factor secretion during biguanide therapy. Diabetologia, 24, 16-18. doi:10.1007/BF00275941

[4] Wulffelé, M.G., Kooy, A., Lehert, P., et al. (2003) Effects of short-term treatment with metformin on serum concentrations of homocysteine, folate and vitamin B12 in type 2 diabetes mellitus: A randomized, placebo-controlled trial. Journal of Internal Medicine, 254, 455-463. doi:10.1046/j.1365-2796.2003.01213.x

[5] Hoogeveen, E.K., Kostense, P.J., Jakobs, C., et al. (1997) Does metformin increase the serum total homocysteine level in non-insulin-dependent diabetes mellitus? Journal of Internal Medicine, 242, 389-394. doi:10.1046/j.1365-2796.1997.00231.x

[6] Savage, D.G., Lindenbaum, J., Stabler, S.P. and Allen, R.H. (1994) Sensitivity of serum methylmalonic acid and total homocysteine determinants for diagnosing cobala$\mathrm{min}$ and folate deficiencies. American Journal of Medicine, 96, 239-246. doi:10.1016/0002-9343(94)90149-X

[7] Lindenbaum, J., Savage, D.G., Stabler, S.P. and Allen, R.H. (1990) Diagnosis of cobalamin deficiency: II. Relative sensitivities of serum cobalamin, methylmalonic acid, and total homocysteine concentrations. American Journal of Hematology, 34, 99-107. doi:10.1002/ajh.2830340205

[8] Ting, R.Z., Szeto, C.C., Chan, M.H., et al. (2006) Risk factors of vitamin $\mathrm{B}(12)$ de ciency in patients receiving metformin. Archives of Internal Medicine, 166, 19751979. doi:10.1001/archinte.166.18.1975

[9] Pongchaidecha, M., Srikusalanukul, V., Chattananon, A., et al. (2004) Effect of metformin on plasma homocysteine, vitamin B12 and folic acid: A cross-sectional study in patients with type 2 diabetes mellitus. Journal of the Medical Association of Thailand, 87, 780-787.

[10] Carlsen, S.M., Kjøtrød, S., Vanky, E., et al. (2007) Homocysteine levels are unaffected by metformin treatment in both nonpregnant and pregnant women with polycystic ovary syndrome. Acta Obstetricia et Gynecologica Scandinavica, 86, 145-150. doi:10.1080/00016340600855946

[11] Sahin, M., Tutuncu, N.B., Ertugrul, D., et al. (2007) Effects of metformin or rosiglitazone on serum concentrations of homocysteine, folate, and vitamin B12 in patients with type 2 diabetes mellitus. Journal of Diabetes and Its Complications, 21, 118-123. doi:10.1016/i.jdiacomp.2005.10.005 